\title{
CardioVascular and Interventional Radiology
}

O Springer-Verlag New York Inc. 1992

\section{Introduction}

\section{Stents}

The rule of thumb in medicine has always been that a new development needs, more or less, one generation to be generally accepted and applied-the time it takes for the new technology to be invented, tested, and approved. This has been no different for expandable intraluminal stents. It took more than 20 years from Charles Dotter's original publication for stents to start to be more widely used in clinical practice. Who would have thought about the multitude of applications for expandable endoluminal stents a few years ago.

This Part I issue, and the following Part II issue, deal with endoluminal metallic stents and encompass articles with different perspectives, covering topics from the historical overview to the very special application in the vascular and the biliary sys- tem, as well as in the trachea. We have tried to place the use of metallic stents in the context of patient management, rather than just describe their use and effects. It was our aim to convey to you, the reader, the opinion and experience of experts who have been using the four different stents with the greatest number of clinical applications. Although we have tried to keep it brief (not too brief). we hope that this is the type of issue you wanted and that you will find all of the articles valuable.

Erich K. Salomonowitz, M.D. Christoph L. Zollikofer, M.D.

Department of Radiology Kantonsspital Winterthur Winterthur, Switzerland 